\title{
High Resolution Clinometers for Measurement of Roll Error Motion of a Precision Linear Slide
}

\author{
Yuki Shimizu*, Satoshi Kataoka and Wei Gao
}

\begin{abstract}
This paper proposes a new method for measurement of the roll error motion of a slide table in a precision linear slide. The proposed method utilizes a pair of clinometers in the production process of a precision linear slide, where the roll error motion measurement will be carried out repeatedly to confirm whether the surface form errors of slide guideways in the linear slide are sufficiently corrected by hand scraping process. In the proposed method, one of the clinometers is mounted on the slide table, while the other is placed on a vibration isolation table, on which the precision linear slide is mounted, so that influences of external disturbances can be cancelled. An experimental setup is built on a vibration isolation table, and some experiments are carried out to verify the feasibility of the proposed method.
\end{abstract}

Keywords: Linear slide, Clinometer, Rotational error motion measurement

\section{Introduction}

In recent, precision linear slides are playing important roles in various ultra-precision machines, such as ultra-precision machine tools or coordinate measuring machines (CMMs) [1-3], in which both a long stroke on the order of several meters and high positioning accuracy on the order of sub-micron to several-tens nanometer are required. For the achievement of such a high positioning accuracy, a closed-loop control with a sufficient position sensor is required. Therefore, most of the precision linear slides are equipped with position sensors such as linear encoders or laser interferometers [4]. Especially, linear encoders are preferred to be employed in linear slides in terms of their low cost, robustness and a high measurement resolution [5].

According to the Abbe principle [6], a measurement axis of the position sensor is preferred to coincide with the motion axis of a linear slide to prevent a positioning error, which is often referred to as the Abbe error, caused by a yaw error motion of the slide table. However, in most

\footnotetext{
*Correspondence: yuki.shimizu@nano.mech.tohoku.ac.jp Department of Finemechanics, Tohoku University, 6-6-01 Aramaki Aza-Aoba, Aoba-ku, Sendai, Miyagi 9808579, Japan
}

of the cases, mechanical structures of such precision linear slides do not meet the Abbe principle [7-9]; there exist a certain amount of offset between the motion axis of a slide table and the measurement axis of a position sensor, which is referred to as the Abbe offset. The Abbe error is a serious error factor for the precision linear slide because even a small yaw error of the slide stable could cause a large positioning error [10-12]. Figure 1 shows a schematic of a linear slide with a linear encoder. When the linear encoder is employed as the position sensor for the linear slide, the offset exists between the motion axis of the slide table and the measurement axis of the linear encoder. When the Abbe offset is on the order of several-hundred $\mathrm{mm}$, even the yaw error motion of $0.0001^{\circ}$ causes a positioning error on the order of severalhundred $\mathrm{nm}$. Measurement of the yaw error motion of the slide table is therefore an important task, and many efforts have been paid so far $[10,13,14]$. Meanwhile, measurement of the roll error motion is also an important task to achieve further precision positioning of the linear slide, although the requirement is more relaxed than that for the yaw error motion measurement since the roll error motion does not directly influence the position detection by the linear encoder. According to the 


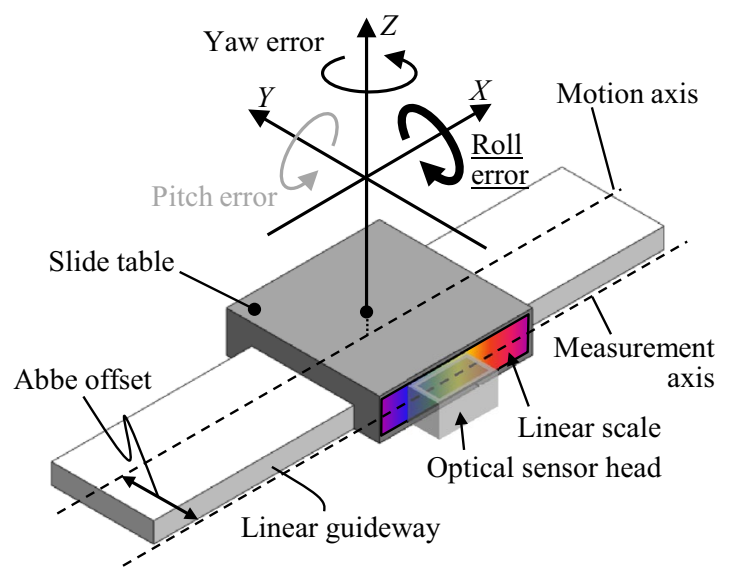

Figure 1 A schematic of tilt error motions of a linear slide

required parallelism in-between the optical sensor head and the grating scale, which is often mounted on the side face of the slide table, the roll error motion of the slide table is required to be less than $0.02^{\circ}$ at least [15].

Rotational error motions of the slide table are induced by the surface form errors of the linear guideways. Therefore, the guideway surfaces are required to be compensated by repeating measurement of the rotational error motions and the form error correction of the guideways in the manufacturing process of linear slides. Although the hand scraping-based error correction is very timeconsuming, it is an essential in the production process of the precision linear slides [16].

For measurement of the yaw error motion, laser interferometers, laser Doppler scales or laser autocollimators [4] have been employed. Especially, autocollimators are often employed in the manufacturing process of linear slides since they can continue their measurement even if their measurement laser beams are interrupted by operators for the hand scraping process. In addition, autocollimators have advantage of high measurement resolution on the order of sub arc-second [17-21]. In the case of measuring the yaw error motion by using an autocollimator, a small mirror reflector will be mounted on a slide table, while an optical sensor head of the autocollimator will be aligned in such a way that the optical axis will be parallel with the motion axis of the slide table. The setup for this measurement is not so difficult since the required size of the reflector is small.

However, on the other hand, measurement of roll error motion requires a long precision mirror, which has the same length as the stroke of the slide. Furthermore, when the stroke of the slide table becomes longer, a disturbance of refractive index in the long optical path influences measurement results. Therefore, it is difficult for a laser autocollimator to carry out measurement of the roll error motion of a linear slide having a long stroke. Meanwhile, a clinometer is another candidate for measurement of the roll error motion. There are three major types of clinometers; the mechanical pendulum-based type [22, 23], the accelerometer-based type [24], and the fluidbased type $[25,26]$. The mechanical pendulum-based clinometers and the accelerometer-based clinometers have advantages of high resolution, large measurement range and small size. Some of those mechanical gravity-based clinometers are designed in a compact size of smaller than $75 \mathrm{~mm} \times 40 \mathrm{~mm} \times 45 \mathrm{~mm}$, while achieving high resolution up to 0.1 arc-second with a good stability [27]. However, they are not suitable for measurement in the manufacturing process of the linear slides since these pendulum-based clinometers have the disadvantages of high cost, and heavy weight owing to its complex mechanism. Meanwhile, on the other hand, the fluidtype clinometers, which detect the direction of the gravity with a liquid surface [28], has the advantages of low cost, small size, and light weight compared with other types of clinometers. In addition, fluid-based type clinometers are robust against external vibration because of its non-mechanical structure. Therefore, the fluid-based type clinometers are suitable to be applied for the manufacturing process of the linear slides. Differing from the autocollimators, the fluid-based type clinometer does not require a mirror reflector because its angle reference is the level of liquid enclosed inside of the sensor body [27, 28]. By utilizing the feature of the clinometer, a setup for measurement of the roll error motion of the slide table in a long-stroke linear slide can therefore be established in a compact size; this is a great advantage from the viewpoint of the production line of the linear slides.

This paper proposes a new measurement method for evaluation of the roll error motion of a precision linear slide by using a high resolution fluid-based type clinometer in the production process of the stage system. The clinometer used in this research is developed based on a commercially-available fluid type clinometer [29], which detects the surface level of the liquid enclosed inside of the sensor body. Since the clinometer detects the absolute angle with respect to the direction of gravitational vector, clinometer detects not only the roll error motion of a slide table but also external inclination such as tilt angle of a vibration isolation table where the linear slide is mounted. In order to remove the influence of the external inclinations, in this paper, another clinometer is directly mounted on the vibration isolation table. By taking a differential output signal of the two clinometers, the roll error motion of the slide table can be evaluated, while eliminating the influence of the external disturbances mainly from the vibration isolation table. 
An experimental setup employing the pair of clinometers is developed, and experiments are carried out to verify the feasibility of the proposed method.

\section{Principle}

Figure 2 shows a schematic of the clinometer and a diagram of its electrical circuit employed in this paper. The clinometer mainly consists of a metal casing, a pair of electrodes and dielectric liquid (paraffin) filling a half of the inner volume of the clinometer. Each electrode is placed to face the metal casing surface with a certain amount of gap $d$. Since a half of the inner volume of the clinometer is filled with the dielectric liquid, the electrodes have capacitance $C$, which is a function of the effective area of the electrodes $S$ and can be described as follows:

$$
C=\varepsilon_{0} \varepsilon_{r} \frac{S}{d}
$$

where $\varepsilon_{0}$ and $\varepsilon_{\mathrm{r}}$ are the permittivities in a vacuum condition and the dielectric liquid, respectively. In Eq. (1), all of the parameters except $S$ are constant. The capacitance $C$ increases in proportion to the effective area $S$ since the fan-shaped electrodes are employed. The effective area $S_{1}$ and $S_{2}$ of the electrodes 1 and 2, respectively, are functions of the rotational angle $\theta$, and can be described by the following equations:

$$
\begin{aligned}
& S_{1}=(\phi / 2-\theta)\left(r_{2}-r_{1}\right) \\
& S_{2}=(\phi / 2+\theta)\left(r_{2}-r_{1}\right)
\end{aligned}
$$

where $r_{1}$ and $r_{2}$ are inner and outer radii of the fanshaped electrodes, respectively. The parameter $\phi$ is the opening angle of the electrodes. Since the electrodes are arranged symmetrically in the sensor structure, the capacitances $C_{1}, C_{2}$ of the electrodes 1 and 2, respectively, can be described by the following equations:

$$
\begin{aligned}
& C_{1}=\varepsilon_{0} \varepsilon_{r} \frac{(\phi / 2-\theta)\left(r_{2}-r_{1}\right)}{d} \\
& C_{2}=\varepsilon_{0} \varepsilon_{r} \frac{(\phi / 2+\theta)\left(r_{2}-r_{1}\right)}{d}
\end{aligned}
$$

The tilt angle $\theta$ about the $X$-axis can be derived by using Eqs. (4) and (5) as follows:

$$
\theta=\frac{d\left(C_{2}-C_{1}\right)}{2 \varepsilon_{r} \varepsilon_{0}\left(r_{2}-r_{1}\right)}=K\left(C_{2}-C_{1}\right)
$$

where $K$ is a constant determined by some parameters such as geometric dimensions of the electrodes in the clinometer, and can be acquired by carrying out calibration with an appropriate calibration standard. Therefore, $\theta$ can be acquired by measuring $C_{1}$ and $C_{2}$, which can be accomplished by using electrical circuits of the clinometers. It should be noted that the clinometer is insensitive to the tilt angle about the $Y$-axis (pitch angle) in Figure 2 because a pair of fan-shaped electrodes is arranged bilaterally symmetrical to the center of the sensor. When the pitch angle is applied to the clinometer, the changes of the effective areas $\Delta S_{1}$ and $\Delta S_{2}$ for the electrodes 1 and 2, respectively, are equivalent due to the symmetrical design of the electrodes. For this reason, capacitance changes $\Delta C_{1}$ and $\Delta C_{2}$ for the electrodes 1 and 2, respectively, are equivalent as well. Since $\theta$ will be calculated by using Eq. (6), in which the changes of the capacitances $\Delta C_{1}$ and $\Delta C_{2}$ will be cancelled, the pitch angle does not affect the detection of $\theta$. It should also be noted that the clinometer is also insensitive against the rotational motion about the $Z$-axis (Yaw angle) in Figure 2, since the effective areas $S_{1}$ and $S_{2}$ will not be affected by the rotation about the $Z$-axis. As described above, the clinometer is insensitive

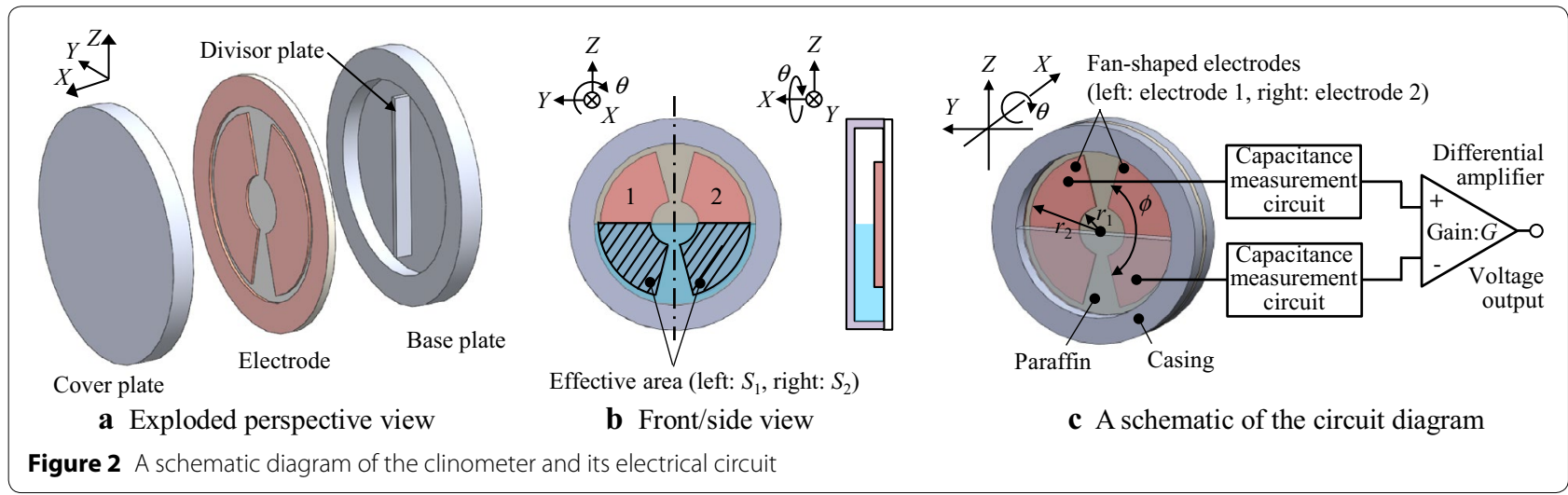


to the pitch and yaw angles; this feature makes the clinometers suitable for measurement of the slide roll error motion. The electrical circuit of the clinometer consists of a trigger pulse generator, a one-shot timer and differential amplifiers. When trigger pulses are provided from the generator, the one-shot timer generates the pulse signals having a pulse width in proportion to the capacitance change. Figure 3 shows a timing chart of signals from the trigger pulse generator and the one-shot timer. The pulse signals are smoothed by passing through a lowpass filter. As a result, voltage signals whose cycles are proportional to the capacitance change can be obtained.

Figure 4 shows a schematic of the setup for measurement of the roll error motion of a linear slide. A pair of clinometers is mounted on a slide table in such a way that the measurement axis of the clinometers is parallel with the motion axis of the slide table. As mentioned before, the clinometers are sensitive to the roll error motion (Figure 5) but insensitive to the pitch and yaw error motions of the slide table. Meanwhile, not only roll error motion of the slide table but also external disturbances such as the tilt angles of the vibration isolation table will be included in the clinometer output. A new method is therefore proposed in this paper to evaluate the roll error motion of the slide table, while removing the influence of the external disturbances. In the method, an additional clinometer is mounted on the vibration isolation table for compensation of the external disturbances. The clinometer mounted on the linear slide is referred to as Sensor 1, while the other directly mounted on the vibration isolation table is referred to as Sensor 2 in this paper. When the roll error motion of the slide table at the $X$-position of $x$ is denoted by $\theta_{\text {Table }}(x)$, the Sensor 1 output $m_{1}(x, t)$ can be expressed by the following equation:

$$
m_{1}(x, t)=\theta_{\text {Table }}(x)+\varphi_{\text {error }}(x, t)
$$

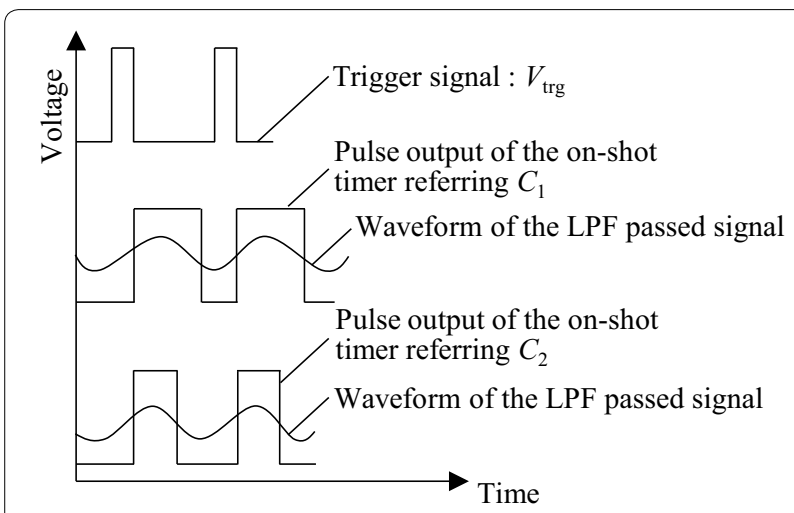

Figure 3 A timing chart of the signals from the trigger pulse generator and the one-shot timer

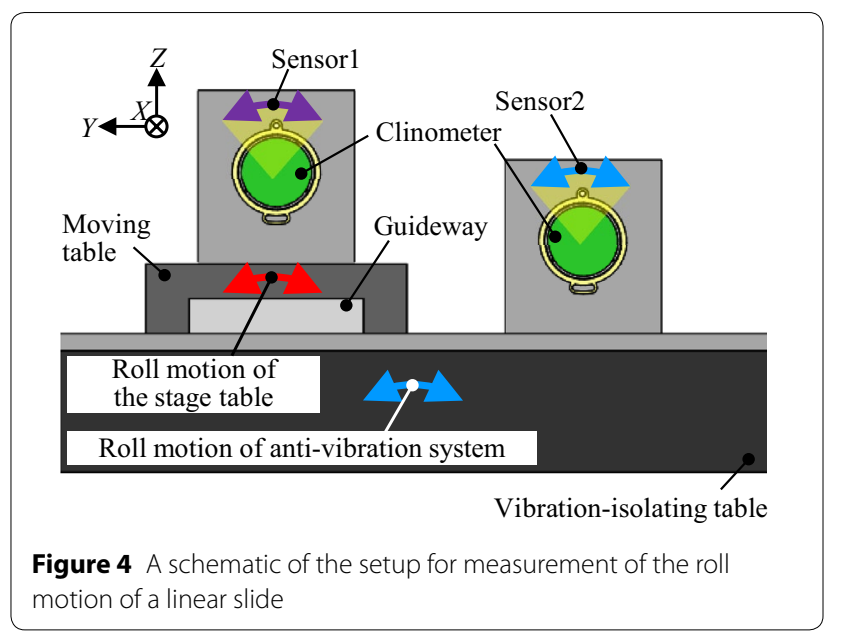

where $\phi_{\text {error }}(x, t)$ is the external disturbances at time $t$. It should be noted that the influence of the AC component of the external disturbances about the $X$-axis can be reduced by taking an average of the Sensor 1 output measured several times at the same $X$-position. However, on the other hand, the influence of the DC component of the external disturbances about the $X$-axis, which could vary in accordance with the position of the slide table $(x)$, still remains in the sensor outputs. To remove the influence of the external disturbances, subtract the Sensor 2 output from the Sensor 1 output. The Sensor 2 output $m_{2}(x, t)$ can be described as follows:

$$
m_{2}(x, t)=\varphi_{\text {error }}(x, t)
$$

By subtracting the Sensor 2 output from the Sensor 1 output, $\theta_{\text {Table }}(x)$ can be acquired as follows:

$$
\theta_{\text {Table }}(x)=m_{1}(x, t)-m_{2}(x, t)
$$

With this simple operation, the influence of the external disturbances can be removed, in principle.

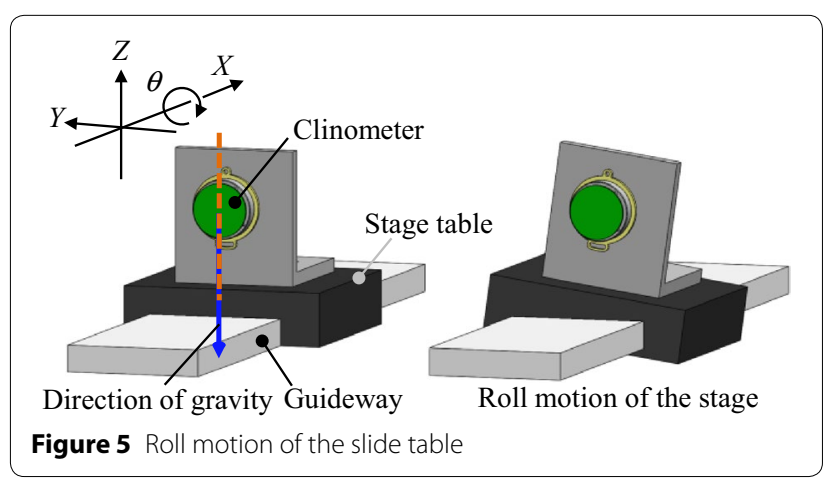




\section{Experiments}

\subsection{Calibration of the Clinometers}

Original angular position of each clinometer used in this paper is determined as the position where the two capacitances $C_{1}$ and $C_{2}$ are balanced. In most of the cases, the clinometers are calibrated during its manufacturing process so that $C_{1}$ and $C_{2}$ would be balanced on the level plane. Meanwhile, for precision angle measurement by the clinometers, their sensitivities should also be calibrated since the linearity of the sensor output is approximately $0.1^{\circ}[29$, which can lead to a large measurement error component in terms of the measurement accuracy required for measurement of slide rotational error motions. In this paper, a precision air-bearing spindle system is therefore employed to calibrate the pair of clinometers. Figure 6(a) and (b) show a schematic and a photograph of the calibration system developed in this paper, respectively. The whole system was built on a vibration isolation system. In the setup, a precision air spindle with the positioning accuracy of $0.0003^{\circ}$ was employed. In the air spindle, a rotary encoder with the measurement resolution of $2.4 \times 10^{-6_{0}}$ was integrated so that the angular position of a rotary table of the spindle could be controlled in a closed loop. Each clinometer was mounted on the rotary table of the precision air spindle in such a way that the measurement axis of the clinometers was aligned to coincide with the rotational axis of the spindle. A voltage output of each clinometer, which varies in accordance with the given angular displacement, was captured by a personal computer.

At first, sensitivity of the Sensor 1 was evaluated. The Sensor 1 was mounted on the rotary table of the spindle, and its angular displacement $\left(\theta_{X}\right)$ was increased step by step. The step increase of $\theta_{X}$ was set to be $0.0001^{\circ}$. According to Eq. (7) in the previous section, the Sensor 1 output will be affected by the external disturbances mainly from the vibration isolation table, which has a resonant frequency on the order of several Hz. Therefore, to eliminate the influence of the external vibration, the rotary table was kept stationary for $120 \mathrm{~s}$ at each angular position, and the averaging of the sensor output was carried out. The sensor output was sampled with the time interval of $1.25 \mathrm{~s}$, and the averaged sensor output over the term from $10 \mathrm{~s}$ to $110 \mathrm{~s}$ was employed as the measured value at each $\theta_{X}$. In the same manner, sensitivity of the Sensor 2 was also calibrated. Figure 7(a) and (b) show the results. In the figures, the horizontal axes indicate $\theta_{X}$ verified by the rotary encoder integrated in the air bearing spindle. According to the slope of each sensitivity curve, sensitivities of Sensor 1 and Sensor 2 were evaluated to be $4.58 \mathrm{~V} /\left(^{\circ}\right)$ and $3.05 \mathrm{~V} /\left(^{\circ}\right)$, respectively, as well as their linearity errors of within $0.001^{\circ}$.

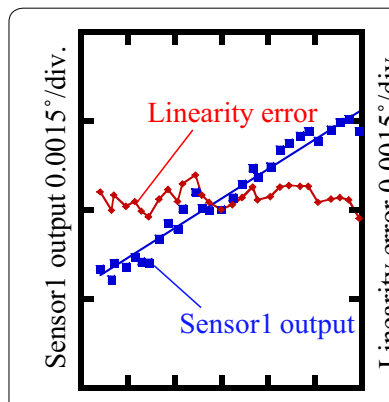

Inclination angle $0.0005 \%$ div a Sensor 1

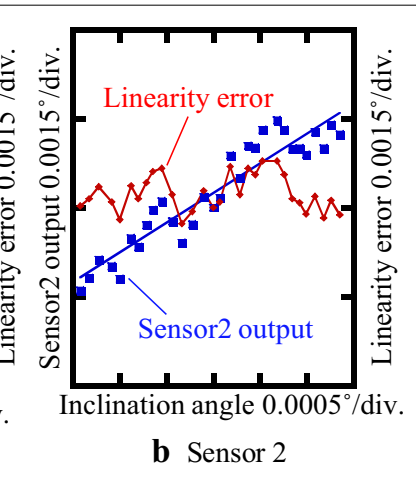

b Sensor 2
Figure 7 Sensitivities of the clinometers

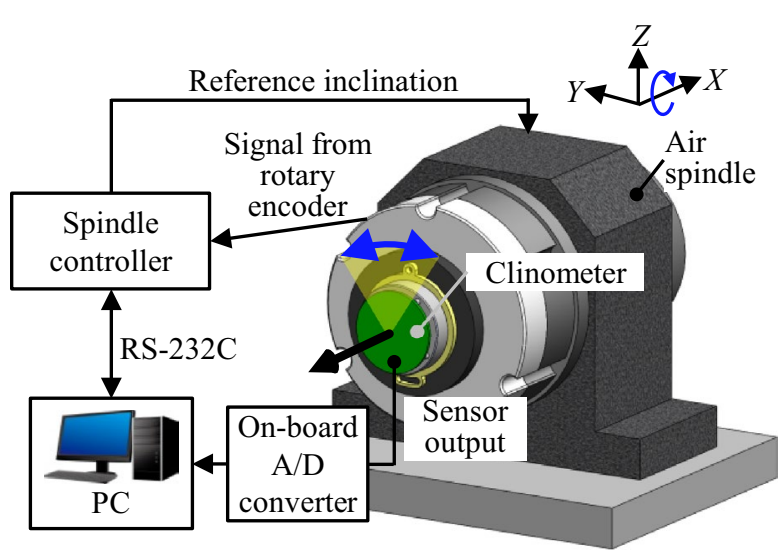

a A schematic of the setup

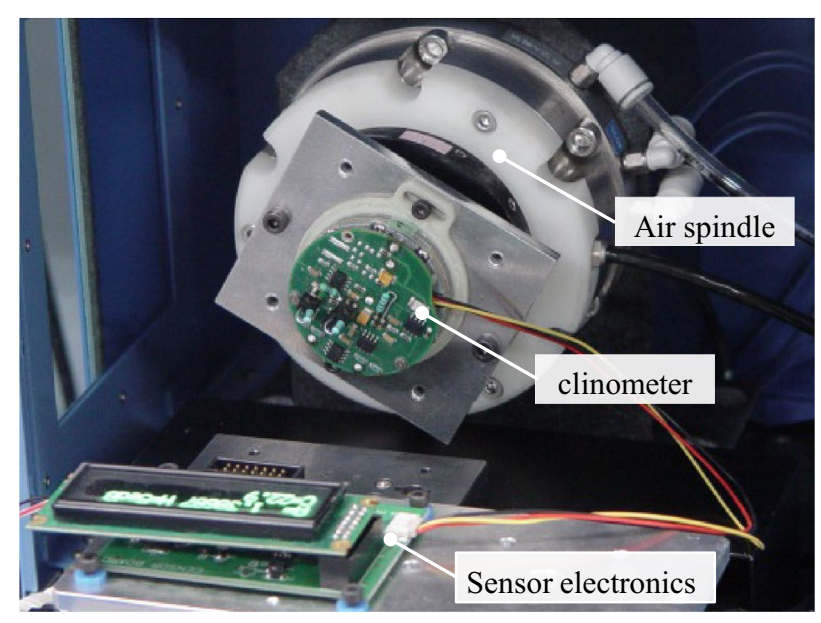

b A photograph of the setup

Figure 6 Calibration system for the clinometers 
After that, the variation of the differential output of the clinometers due to the change of $\theta_{X}$ was investigated. Figure $8(\mathrm{a})$ and (b) show a schematic and a photograph of the experimental setup used for this experiment, respectively. In this experiment, two clinometers were stacked and mounted on a tilt stage so that the inclination angle applied to the two clinometers would be equivalent. During the experiment, the vibration isolation table was kept stationary. In the experiments, the inclination angles with a step of $0.005^{\circ}$ were given to the tilt stage. At each step of the stage motion, the stage was kept stationary over a term of $150 \mathrm{~s}$. The sensor outputs were sampled with a frequency of $0.8 \mathrm{~Hz}$. The data taken over the term from $50 \mathrm{~s}$ to $150 \mathrm{~s}$ was used to obtain an averaged angular output. Figure 9 shows the result. The horizontal and vertical axes shows the reference inclination angle and the angular output, respectively. From the experimental results, the variation of differential output is found to be within $0.0015^{\circ}$.

In addition, to verify the feasibility of the proposed method, external disturbances were intentionally given to the measurement system by tilting the vibration isolation table, on which the clinometers were mounted. Figure 10(a) and (b) show a schematic and a photograph of the prepared experimental setup, respectively. A laser autocollimator (Möeller-Wedel Optical, Elcomat 3000) was also mounted on the vibration-isolation table as a reference for measurement of the stage roll error motion. The external inclination angle of approximately $0.02^{\circ}$ was given to the entire measurement system every $60 \mathrm{~s}$. It should be noted that the slide table of the linear slide was kept stationary during the experiment. Figure 11 shows the results. In the figure, the horizontal axis shows the measurement time, while the vertical axis shows the outputs from the clinometers and laser autocollimators. The differential output of the clinometers is also plotted in the figure. The autocollimator output was constant because the relative position of the reflector with respect to the laser autocollimator, both of which were mounted on the same vibration isolation table, did not change. However,

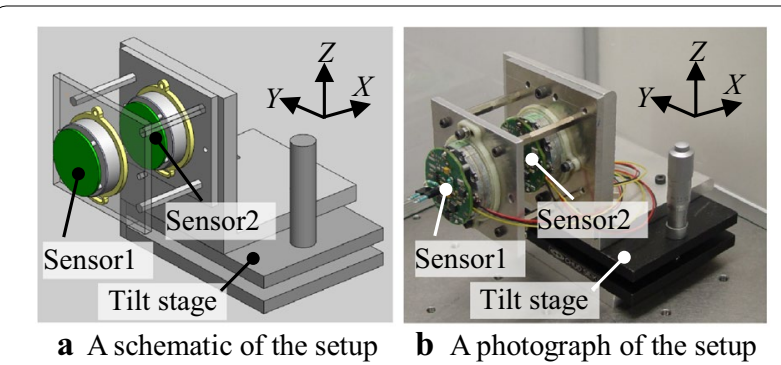

Figure 8 A setup for evaluation of sensor linearity

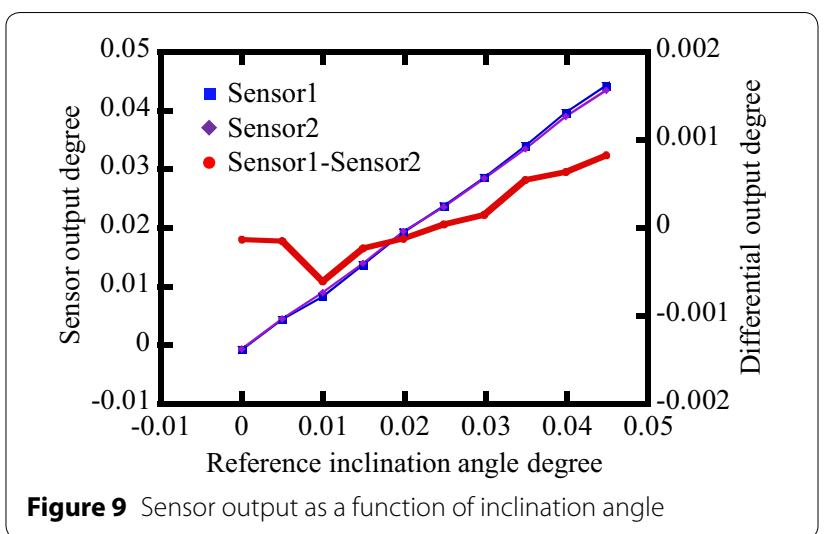

on the other hand, five peaks can be found in the differential output signal of the Sensor 1 and Sensor 2. The cause of the peaks is considered to be the calibration errors in the sensitivity of the clinometers. Meanwhile, the differential signal output was found to be stable soon after applying the inclination angle to the vibration isolation table. Therefore, the proposed method is expected to be valid when the slide table of the linear slide is kept stationary.

\subsection{Roll Error Measurement for a Linear Slide}

By using the calibrated clinometers, the roll error motion of the precision linear slide was then evaluated

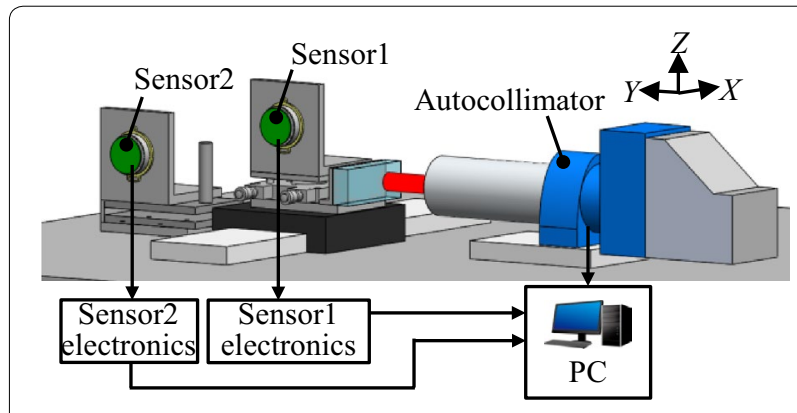

a A schematic of the experimental setup

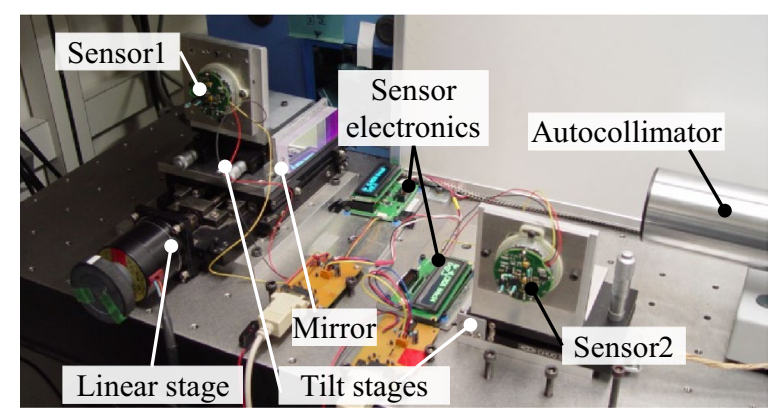

b A photograph of the experimental setup

Figure 10 A setup for stage roll error measurement 


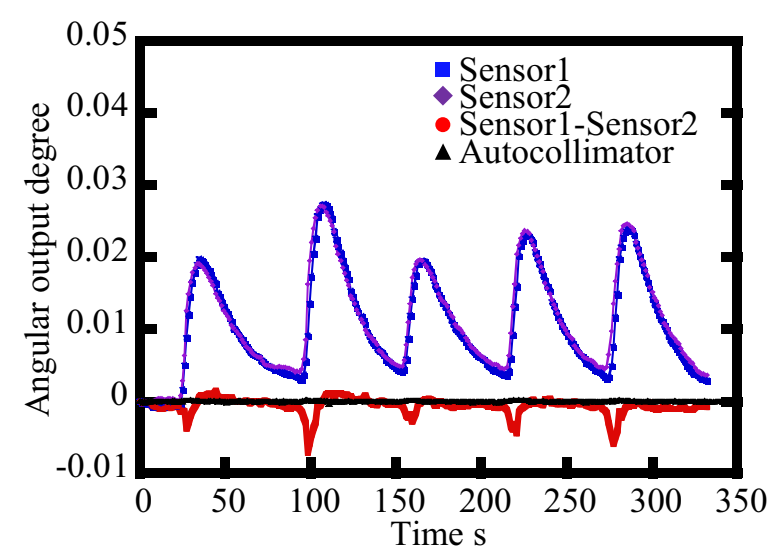

Figure 11 Variations of sensor outputs due to external disturbance

in experiments. The setup shown in Figure 10 was also employed in the experiments. As shown in the figure, the Sensor 1 was mounted on the slide table, while the Sensor 2 was directly fixed on the vibration isolation table. Since the measurement range of each clinometer was set to be small for enhancement of the measurement resolution, Sensor 1 and 2 were mounted on different manual tilt stages independently. The stage roll error motion was evaluated by using both the developed clinometers and the commercial laser autocollimator, which was employed as a reference in the experiments. Since the commercial autocollimators cannot measure roll motion of a linear slide with a long stroke due to the absence of a precision mirror having a length comparable with the slide, a linear stage with a short stroke of $45 \mathrm{~mm}$ was employed in this paper. A precision reflective mirror with the length of $100 \mathrm{~mm}$ was also mounted on the slide table so that slide roll motion could be measured by the commercial laser autocollimator.

At first, stability of the experimental setup was evaluated. Variations of the sensor outputs were monitored with the sampling frequency of $1.25 \mathrm{~Hz}$, while the slide table was kept stationary. Figure 12 shows the results. Variation of the room temperature measured simultaneously is also plotted in the figure. It should be noted that the vibration isolation table was kept stationary to eliminate the influences of external disturbances. The output of the each clinometer varied within a range of approximately $0.002^{\circ}$ in the measurement. It was also verified that the variation amplitude of the differential output of the two clinometers was in the same range. In general, stability of the sensor output is affected by the temperature variation. If the measurement term is shorter than $1500 \mathrm{~s}$, the temperature variation is reduced by the part. Thus, it has been verified that the

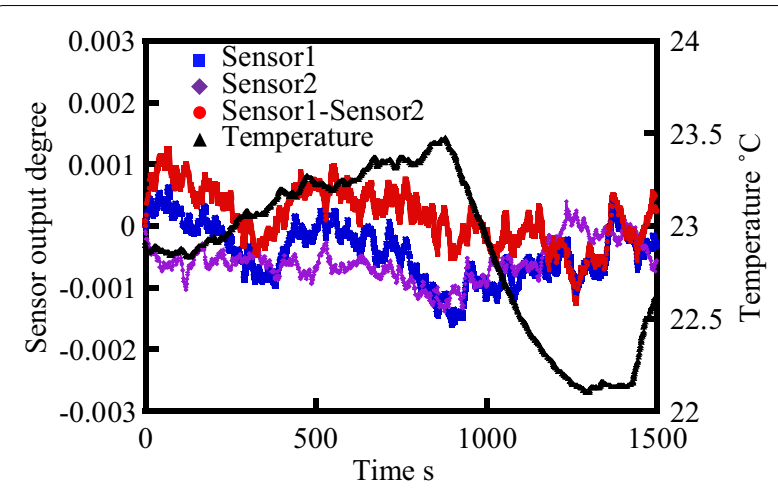

Figure 12 Stability of the sensor outputs

measurement system prepared in this paper is expected to have stability better than $0.002^{\circ}$ in the measurement term of $1500 \mathrm{~s}$.

Following the stability measurement, slide roll motion was measured by using the developed measurement system. In the experiment, the slide table of the linear slide was moved from its original position to the $X$-position of $45 \mathrm{~mm}$. The slide table was kept stationary at each $5 \mathrm{~mm}$ in the travel range for $180 \mathrm{~s}$, and its roll error motion was measured by using both the clinometers and the laser autocollimator. Each sensor output captured over the term from $50 \mathrm{~s}$ to $150 \mathrm{~s}$ was averaged, and the averaged value at each $X$-position was plotted in the figure. Sampling frequencies of the clinometers and the laser autocollimator were set to be $1.25 \mathrm{~s}$ and $0.4 \mathrm{~s}$, respectively. Figure 13 shows the results. In the figure, the horizontal and vertical axes show the table displacement and the measured roll error motion, respectively. A good agreement can be found between the laser autocollimator output and the differential output signal of the Sensors 1 and 2. From these results, it has been verified that the influence of the external inclination has successfully been eliminated from the Sensor 1. Since the developed clinometer does not require a reference such as a precision mirror or a precision straightedge, which is mandatory for the laser autocollimators or laser interferometers, the proposed method is also effective in measuring the roll error motion of a linear slide having a long stroke. Verification test of the proposed method in the production line of a linear slide, and the investigation on the measurement uncertainty of the proposed method will be carried out as future work.

\section{Conclusions}

For measurement of roll error motion of a slide table in a precision linear slide during its manufacturing process, a new measurement method using a pair of clinometers 


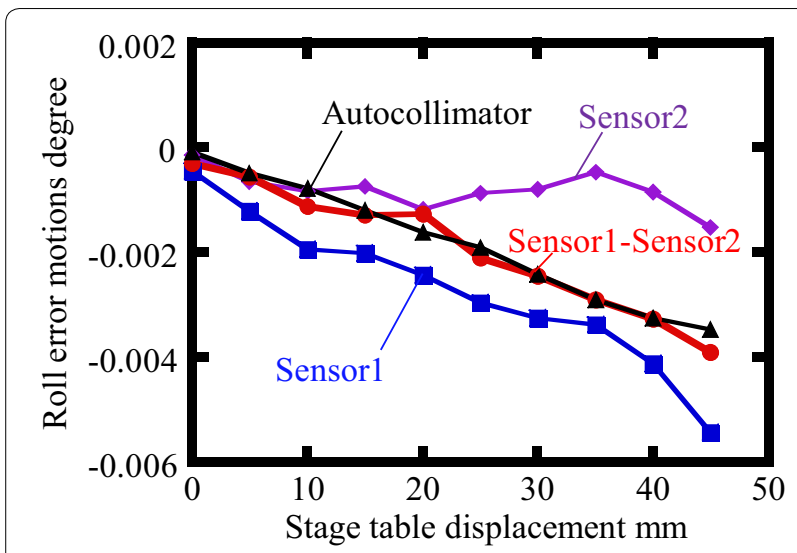

Figure 13 Roll error motion measured by the developed system

has been proposed, and its feasibility has been verified in experiments. At first, the sensitivities of the clinometers have been calibrated by using the precision air spindle having a precision rotary encoder. The linearity error of each sensor has been verified to be within $0.001^{\circ}$. The differential output of the two clinometers has also been calibrated, and the output difference between the two clinometers has been verified to be within $0.0015^{\circ}$. Finally, the roll error motion of the slide table has been evaluated by using the proposed method. By taking the differential of the sensor outputs, the roll error motion of the slide has successfully been measured, while eliminating the influence of the external disturbances.

\section{Authors' Contributions}

YS was in charge of the whole trial; SK assisted with sampling and laboratory analyses; and YS and WG wrote the manuscript. All authors read and approved the final manuscript.

\section{Authors' Information}

Yuki Shimizu received his bachelor and MS degrees from Tohoku University, Japan, in 2000 and in 2002, respectively. He received his PhD degree from Department of Mechanical Engineering, Nagoya University, Japan, in 2009. He is currently an associate professor at Nano-Metrology and Control Laboratory, Department of Finemechanics, Tohoku University, Japan.

Satoshi Kataoka is currently a master candidate at Nano-Metrology and Control Laboratory, Department of Finemechanics, Tohoku University, Japan.

Wei Gao received his bachelor degree in precision engineering from Shanghai Jiao Tong University, China, in 1986 and MS and PhD degrees in engineering from Tohoku University, Japan, in 1991 and 1994, respectively. He is currently a professor and a director of Research Center for Precision Nanosystems, Department of Finemechanics, Tohoku University, Japan. His research interests include optical sensors, precision dimensional metrology and motion control. He is a member of JSPE, JSME, CIRP and a Fellow of The International Society for Nanomanufacturing. He is serving as the Chairman of The Scientific Technical Committee Precision Engineering and Metrology of CIRP. He is the author of the book Precision Nanometrology published by Springer.

\section{Acknowledgements}

This project was supported by Japan Society for the Promotion and Science (JSPS). The authors would like to thank Mr. Satoshi Nakagawa for his valuable comments and suggestions on our experiments.

\section{Competing Interests}

The authors declare that they have no competing interests.

\section{Publisher's Note}

Springer Nature remains neutral with regard to jurisdictional claims in published maps and institutional affiliations.

Received: 18 April 2018 Accepted: 22 October 2018

Published online: 01 November 2018

\section{References}

[1] K Sato. High-precision and high-speed positioning of $100 \mathrm{G}$ linear synchronous motor. Precision Engineering, 2015, 39: 31-37.

[2] M Week. Linear magnetic bearing and levitation system for machine tools, CIRP Annals-Manufacturing Technology, 1998, 47(1): 311-314.

[3] K Erkorkmaz, M Gorniak. Precision machine tool X-Y stage utilizing a planar air bearing arrangement. CIRP Annals-Manufacturing Technology, 2010, 59(1): 425-428.

[4] H Kunzmann, T Pfeifer, J Flügge. Scales vs. laser interferometers performance and comparison of two measuring systems. CIRP Annals-Manufacturing Technology, 1993, 42(2): 753-767.

[5] W Gao, SW Kim, H Bosse, et al. Measurement technologies for precision positioning. CIRP Annals-Manufacturing Technology, 2015, 64(2): 773-796.

[6] J B Bryan. The Abbe principle revisited - an updated interpretation. Precision Engineering, 1979, 1(3): 129-132.

[7] W Knapp. Measurement uncertainty and machine tool testing. CIRP Annals-Manufacturing Technology, 2002, 51(1): 459-462.

[8] H Schwenke, W Knapp, H Haitjema, et al. Geometric error measurement and compensation of machines - an update. CIRP Annals-Manufacturing Technology, 2008, 57(2): 660-675.

[9] Y Shimizu, S Goto, S Ito, et al. Fabrication of large-size SiC mirror with precision aspheric profile for artificial satellite. Precision Engineering, 2013, 37: 640-649.

[10] W Gao, Y Arai, A Shibuya, et al. Measurement of multi-degree-of-freedom error motions of a precision linear air-bearing stage. Precision Engineering, 2006, 30(1): 96-103.

[11] W Gao. Precision nanometrology. Springer, London, 2010.

[12] X Li, W Gao, H Muto, et al. A six-degree-of-freedom surface encoder for precision positioning of a planar motion stage. Precision Engineering, 2013, 37: 771-781.

[13] K C Fan, M J Chen. A 6-degree-of-freedom measurement system for the accuracy of X-Y stages. Precision Engineering, 2000, 24(1): 15-23.

[14] S L Tan, Y Shimizu, T Meguro, et al. Design of a laser autocollimator-based optical sensor with a rangefinder for error correction of precision slide guideways. International Journal of Precision Engineering and Manufacturing, 2015, 16(3): 423-431.

[15] Heidenhain. Exposed linear encoders. Heidenhain, 2016.

[16] W R Moore. Foundations of mechanical accuracy. The Moore Special Tool Co., MIT Press, 1970

[17] W Gao, Y Saito, H Muto, et al. A three-axis autocollimator for detection of angular error motions of a precision stage. CIRP Annals-Manufacturing Technology, 2011, 60(1): 515-518.

[18] Y Shimizu, S L Tan, D Murata, et al. Ultra-sensitive angle sensor based on laser autocollimation for measurement of stage tilt motions. Optics Express, 2016, 24(3): 2788-2805.

[19] Y L Chen, Y Shimizu, Y Kudo, et al. Mode-locked laser autocollimator with an expanded measurement range. Optics Express, 2016, 24(14): 15554-15569.

[20] Y L Chen, Y Shimizu, J Tamada, et al. Optical frequency domain angle measurement in a femtosecond laser autocollimator. Optics Express, 2017, 25(14): 16725-16738.

[21] J Tamada, Y Kudo, Y L Chen, et al, Determination of the zero-position for an optical angle sensor. Journal of Advanced Mechanical Design, Systems, and Manufacturing, 2016, 10(5): 00072.

[22] K C Fan, T H Wang, SY Lin, et al. Design of a dual-axis optoelectronic level for precision angle measurements. Measurement Science and Technology, 2011, 22(5): 055302. 
[23] K Venkateswara, C A Hagedorn, M D Turner, et al. A high-precision mechanical absolute-rotation sensor. Review of Scientific Instruments, 2014, 85(1): 015005.

[24] F S Alves, R A Dias, J M Cabral, et al. High-resolution MEMS inclinometer based on pull-in voltage. Journal of Microelectromechanical Systems, 2015, 24(4): 931-939.

[25] H Ueda, H Ueno, K Itoigawa, et al. Development of micro capacitive inclination sensor. IEEJ Transactions on Sensors and Micromachines, 2006, 126(12): 637-642.

[26] Y Shimizu, S Kataoka, T Ishikawa, et al. A liquid-surface-based three-axis inclination sensor for measurement of stage tilt motions. Sensors, 2018, 18: 398 .
[27] Sherbome Sensor, LSOP-LSOC-2013 Iss1. Sherborne Sensors, 2013.

[28] S L Tan, S Kataoka, T Ishikawa, et al. An ultra-precision electronic clinometer for measurement of small inclination angles. Journal of the Korean Society of Manufacturing Technology Engineers, 2014, 23(6): 539-546.

[29] S Kataoka, T Ishikawa, Y Shimizu, et al. Measurement of angular error motions of a precision linear stage by using a high resolution clinometer. Proceedings of the 8th International Conference on Leading Edge Manufacturing in 21st Century, 2015: https://doi.org/10.1299/jsmel em.2015.8._1505-1_.

\section{Submit your manuscript to a SpringerOpen ${ }^{\odot}$ journal and benefit from:}

- Convenient online submission

- Rigorous peer review

- Open access: articles freely available online

- High visibility within the field

- Retaining the copyright to your article

Submit your next manuscript at $\boldsymbol{\nabla}$ springeropen.com 